\title{
Comparative studies on molecular techniques for detecting Rickettsia tsutsugamushi in Leptotrombidium deliense and mice
}

\author{
ZHIYONG XI, JIACAN LI, XIAOYING ZHENG \& XIE YAN \\ Department of Parasitology, Sun Yat-sen University of Medical Sciences, Guangzhou 510089, China
}

\begin{abstract}
In order to establish the most sensitive and specific method in the detection of Rickettsia tsutsugamushi in Leptotrombidium deliense, mice and patients, a comparative study on DNA extraction, PCR, hybridization and PCR-hybridization was conducted. The results showed that the sensitivity of PCR-hybridization was 10 times higher than that of PCR and 1000 times higher than that of hybridization. Quick DNA extraction method could take the place of phenol-chloroform method to prepare DNA template when detecting the rickettsiae in mice and patients' blood by PCR. De-inhibitor quick DNA extraction method could improve the detection rate of rickettsiae from chigger mites.
\end{abstract}

Key words: Leptotrombidum deliense, Rickettsia tsutsugamushi, PCR, hybridization, PCR-hybridization

\section{Introduction}

Scrub typhus is a natural epidemic disease spread by the larval chiggers of Leptotrombidium (Leptotrombidium) deliense (Walch), L.(L.) arenicola (Traub) and L.(L.) fletcheri Vercammen-Grandjean (Daryl et al. 1994). In nature, both mice and chiggers can be the reservoir hosts of $R$. tsutsugamushi. Our focus is on the cycling mechanism of Rickettsia tsutsugamushi (Ogata) between chigger mites and mice, and its spread potential. Traditional methods (including animal infection isolating method, immune method and eletronmicroscopy in detection of the rickettsiae) have their own disadvantages (Hong et al. 1991). Thus molecular techniques for detecting R. tsutsugamushi have been developed (Ni et al. 1995; Li et al. 1994). In order to establish a most sensitive and specific method for the detection of $R$. tsutsugamushi in chiggers and mice, a comparative study on DNA extraction, PCR, hybridization and PCR-hybridization was carried out.

\section{Materials and methods}

Mites

A pure line of $L$. deliense routinely cultured in our laboratory was confirmed free of the rickettsial infection by purification tests with different mite stages including larvae, nymphs, preadults and adults.

Chiggers infected with $R$. tsutsugamushi were obtained either by inoculating $R$. tsutsugamushi into the abdomen of normal adults or by allowing mite larvae to bite infected mice, and then culture these mites. Different mite stages were accumulated for use in experiments. 


\section{Mice blood and spleen}

Pure line BALB/C mice, which was supplied by the Animal Centre of Sun Yat-sen University of Medical Sciences, was infected with $R$. tsutsugamushi by abdominal inoculation, or by bite of rickettsiae-infected larvae, and then cultured for three weeks. When the mice became ill, blood and spleen were collected for experiments.

Blood and spleen were also accumulated from pure line BALB/C mice.

\section{Rickettsial strains}

Karp standard strains of $R$. tsutsugamushi were preserved at $-70^{\circ} \mathrm{C}$ in our laboratory since 1991 . Karp DNA was extracted by phenol-chloroform method; serial dilutions of 1:10, 1:10² $1: 10^{3}, 1: 10^{4}$, $1: 10^{5}, 1: 10^{6}$ and $1: 10^{7}$ were prepared with TE buffer ( $10 \mathrm{mM}$ Tris $\cdot \mathrm{Cl}, \mathrm{pH}$ 8.0; $1 \mathrm{mM}$ EDTA, $\left.\mathrm{pH} 8.0\right)$.

Rickettsia prowazekii (da Rocha-Lima), R. mooseri (Monterio), R. sibericus (Zdrodovskii) and Gilliam strain of R. tsutsugamushi were provided by the Guangdong Sanitation and Anti-epidemic Station. Kato strain of $R$. tsutsugamushi was also preserved by our laboratory. By phenol-chloroform method, DNA of all these samples were prepared.

\section{Patient blood pool}

Rickettsiae-infected blood samples from patients were provided by the Department of Paediatrics and the Department of Infectious Diseases, Fushan First Hospital of Guangdong Province. All patients had typical symptoms and signs of scrub typhus characterized by fever and escharosis.

\section{Primers}

Four primers were designed from Sta56 gene of $R$. tsutsugamushi and synthesized by Biomedical Inc.(Alambra, USA)

1. 5'-TTGCTAGTGCAATGTCTGC-3';

2. 5'-CCCTGCTGCTGTGCTTGCTGCCG-3';

3. 5'-TCCTCAGCCTACTATAATGCC-3';

\section{5'-CGACAGATGCAACTATTAGGC-3'.}

Amplification fragment was $1 \mathrm{~kb}$ when PCR was performed with primers 1 and 2. Amplification fragment was $500 \mathrm{bp}$ when PCR was performed with primers 3 and 4.

\section{Molecular techniques}

DNA extraction: Three DNA extraction methods were adopted: classic phenol-chloroform method, quick DNA extraction method and chiggers de-inhibition quick DNA extraction method. Quick DNA extraction liquid was prepared by the senior author. Chiggers de-inhibition quick DNA extraction method was as follows: chiggers were triturated in quick DNA extraction liquid and then boiled for 10 minutes. Samples were then put on hybridization membrane. After being dried, the membrane was washed with $1 \mathrm{~mol} / \mathrm{L} \mathrm{NaCl}$ and TE (pH 8.0) to remove inhibitor factors. DNA fixed membrane was employed for PCR.

PCR Assay: PCR was performed by a programmable thermal controller (model PTC-150, MJ Inc.) and the DNA Amplification Reagent Kit (Biomedical Inc.) with Taq polymerase, $1 \mathrm{U}$ per reaction in a total $50 \mu \mathrm{l}$ reaction volume. The PCR mixture was denatured at $94^{\circ} \mathrm{C}$ for 30 seconds, annealed at $57^{\circ} \mathrm{C}$ for 1 minute, and then the chain was extended at $70^{\circ} \mathrm{C}$ for 2 minutes. This cycle was repeated 35 times and the sample was then held at $4^{\circ} \mathrm{C}$ overnight. In the case of DNA fixed membrane, it was soaked in PCR reaction mixture for 10 cycles. After that, the membrane was removed and $1 \mathrm{U}$ Taq polymerase was added again, and then PCR was allowed to continue. $2 \mu 1$ of the first 
PCR product (primer 1-2) diluted to 1:10 in TE was used in nest-PCR (primer 3-4). $5 \mu 1$ of the product of nest-PCR was electrophoresed on $1 \%$ agarose gel with ethidium bromide as the indicator and pBR322/Hinf I as DNA markers. After EB staining, the bands were observed under ultraviolet detector (Furuya 1993).

Hybridization Assay: The probe from Sta56 was marked with digoxigenin by PCR. The temperature for hybridization was $68^{\circ} \mathrm{C}$. The hybridization method used follows that in the manual of DIG DNA Labelling and Detection Kit (Boehringer Mannheim Co.).

Detection of PCR products with blotting method (PCR-hybridization): PCR products were put in a water bath at $100^{\circ} \mathrm{C}$ for 5 minutes. They were then transferred to an ice bath. $2 \mu 1$ of the product were dotted and detected.

\section{Results and discussion}

Comparison of three DNA extraction methods for detection of R. tsutsugamushi

$P C R$ results of the classic phenol-chloroform method and quick extraction method: DNA from 20 samples of $R$. tsutsugamushi positive mice blood, 15 samples of positive mice spleen and 15 samples of positive patient blood were extracted with the classic phenol-chloroform method and quick extraction method respectively. The positive rate with quick extraction method was slightly lower than with classic method in mice spleen, but there was no significant difference $(P>0.05)$. Detection rates with the two methods were the same in patient blood and mice blood, which suggested that quick extraction method could be used to prepare the sample (Table 1). Because the duration of the experiment was shortened, it was possible that the detection of $R$. tsutsugamushi with PCR could be applied in clinical diagnosis.

TABLE 1. The results of PCR after Rickettsia tsutsugamushi DNA extraction with phenol-chloroform method and quick extraction method.

\begin{tabular}{cccc}
\hline \multirow{2}{*}{ Samples } & \multirow{2}{*}{ No. of samples } & \multicolumn{3}{c}{ Positive results (\%) } \\
& & Phenol-chloroform method & Quick extraction method \\
\hline Mice blood & 20 & 100.00 & 100.00 \\
Mice spleen & 15 & 100.00 & 93.33 \\
Patient blood & 15 & 100.00 & 100.00 \\
\hline
\end{tabular}

The results of PCR before and after removing inhibitor factors: 30 samples of $R$. tsutsugamushi infected adults, engorged larvae and nymphs of $L$. deliense were selected from laboratory cultures. DNA from each group was extracted with quick extraction method and de-inhibition quick extraction method respectively. It was showed that there was significant difference between the results obtained before and after removal of inhibitory factors (Table 2). The positive rate dramatically increased after removal of inhibitory factors $(\mathrm{P}<0.05)$, suggesting that the de-inhibition quick extraction method could be used to extract DNA from $R$. tsutsugamushi in chiggers. 
TABLE 2. The results of PCR before and after removal of inhibitory factors in Leptotrombidium deliense.

\begin{tabular}{cccc}
\hline \multirow{2}{*}{ Samples } & \multirow{2}{*}{ No. of samples } & \multicolumn{2}{c}{ Positive results (\%) } \\
& & Before removal & After removal \\
\hline Mice blood & 30 & 40.00 & 100.00 \\
Mice spleen & 30 & 26.67 & 96.67 \\
Patient blood & 30 & 33.33 & 93.33 \\
\hline
\end{tabular}

DNA extraction from $\mathrm{R}$. tsutsugamushi in chiggers: Classical DNA extraction methods were deemed unsuitable because of great DNA lost and only trace of $R$. tsutsugamushi in chiggers. On the other hand, the purity of DNA for PCR is not very important. Therefore, the quick extraction method has been recommended by some researchers (Daryl et al. 1994). Because of the presence of inhibitors of Taq polymerase in chiggers after engorgement, it was necessary to remove them when employed for PCR. In this study we performed de-inhibition quick extraction method with success.

Specificity of PCR, hybridization and PCR-hybridization for detecting R. tsutsugamushi

The detection of three international standard strains of R. tsutsugamushi (Karp, Kato and Gilliam), $R$. prowazekii, $R$. mooseri, $R$. sibericus and normal mice blood showed that the three standard strains (Karp, Kato and Gilliam) could be detected by the above three methods, while the other three species of Rickettsiae could not. It was suggested that the three methods could be used to detect $R$. tsutsugamushi in samples (data not shown).

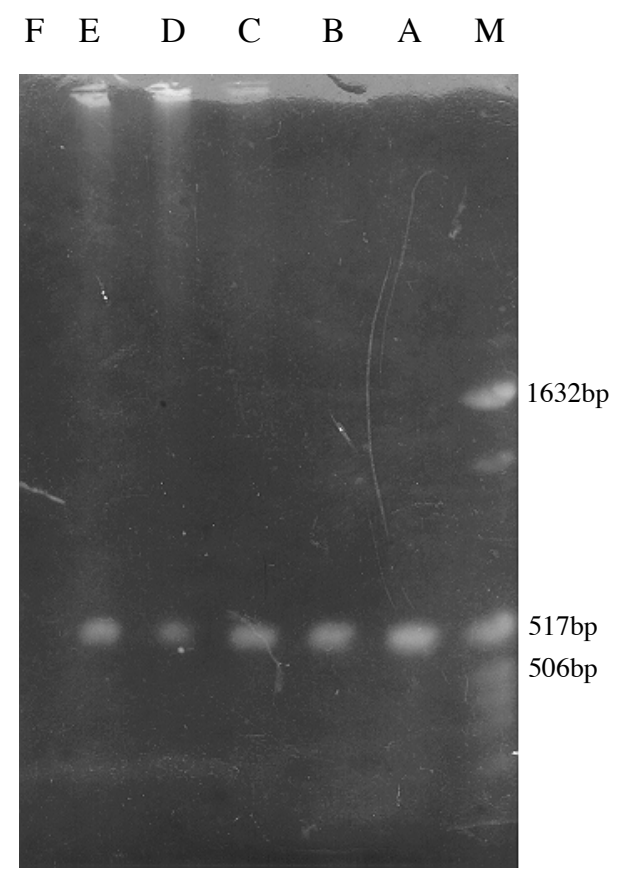

FIGURE 1. Sensitivity of PCR for detecting Rickettsia tsutsugamushi. Serial dilutes of Karp DNA: A. 1:10,

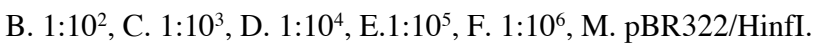


Sensitivity of PCR, hybridization and PCR-hybridization to detect $\mathrm{R}$. tsutsugamushi

Seven serial dilutes of Karp strain DNA were detected by the above three methods. It was demonstrated that the highest diluted concentration which could be detected by PCR was 1:105 (Fig. 1), by hybridization was $1: 10^{3}$ (Fig. 2), by PCR-hybridization was $1: 10^{6}$ (Fig. 3). PCR-hybridization was 10 times as sensitive as PCR alone, and 1000 times more than hybridization. Theoretically, the method of DNA agarose electrophoresis and EB staining is not very sensitive. For a fragment of 100 bp, visible bands appear on the gel only after 1010 amplification. The detection limit is 10 ng DNA by agarose electrophoresis. However, the detection limit is $10 \mathrm{pg}$ when hybridization was used in place of agarose electrophoresis. Moreover, some accidental factors may cause nonspecific amplification in PCR, and the size of the amplified bands is similar to that of target bands. As a consequence, false positive result may occur by electrophoresis, but this can be avoided by detection via probes. Therefore, PCR-hybridization can also improve the specificity for detection of the rickettsiae.

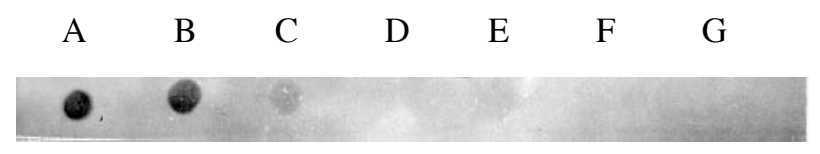

FIGURE 2. Sensitivity of hybridization for detecting Rickettsia tsutsugamushi. Serial dilutes of Karp DNA: A. $1: 10$, B. $1: 10^{2}$, C. $1: 10^{3}$, D. $1: 10^{4}$, E. $1: 10^{5}$, F. $1: 10^{6}$, G. $1: 10^{7}$.

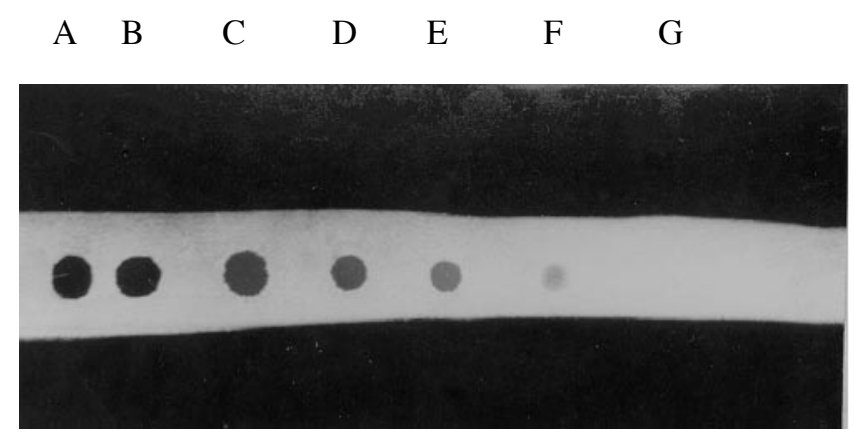

FIGURE 3. Sensitivity of PCR-hybridization to detect Rickettsia tsutsugamushi. Serial dilutes of Karp DNA:

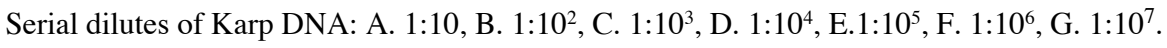

\section{Acknowledgements}

This project was supported by the National Natural Science Foundation of China (Project No. 39370636). We are grateful to Prof. Tinghuan Wen of Medical Acarology Laboratory of Shanghai Medical University for his peer review of this manuscript. 


\section{References}

Daryl, J.K., Gregory, A.D., Chan, T.C. \& Ho, T.M. (1994) Detection and characterization of Rickettsia tsutsugamushi (Rickettsiales: Rickettsiaceae) in infected Leptotrombidium (Leptotrombidium) fletcheri chiggers (Acari: Trombiculidae) with the polymerase chain reaction. Journal of Medical Entomology, 31(5), 691699.

Furuya, Y. (1993) Serotype-specific amplification of Rickettsia tsutsugamushi DNA by nest-PCR. Journal of Clinical Microbiology, 31(6), 1637-1640.

Hong, F., Li , J.C. \& Xi, B.K. (1991) Experimental infection of Leptotrombidium deliense with Rickettsia tsutsugamushi. Chinese Journal of Zoonoses, 7(4), 2-5 [In Chinese].

Li, J.C., Zheng, X.Y., Ni, H. \& Cheng, T.S. (1994) Experimental studies on detection of Rickettsia tsutsugamushi in red- and white-bodied Leptotrombidium deliense by DNA amplification using polymerase chain reaction technology. Chinese Journal of Zoonoses, 10(3), 2-5 [In Chinese].

Ni, H., Li, J.C. \& Cheng,T.S. (1995) Detection of the dynamic state of Rickettsia tsutsugamushi in trombiculids and study on the transovarial transmission of Rickettsia tsutsugamushi. Acta Parasitologica et Medica Entomologica Sinica, 1(2), 48-53 [In Chinese].

Accepted: 20 March 1999 